\title{
Comportamento de modelos adaptativos de conforto térmico frente ao modelo de Fanger em intensivistas adaptados ao clima tropical
}

The behaviour of adaptive thermal comfort models compared to the Fanger model in intensivists adapted to a tropical climate

\section{Elamara Marama Araujo Vieira \\ J onhatan Magno Norte da Silva \\ Wilza Karla dos Santos Leite \\ Manoel Gerônimo Lino Torres Luiz Bueno da Silva}

\section{Resumo}



bjetivou-se investigar o comportamento de modelos adaptativos de conforto térmico (PMVay e PMVak) em relação ao modelo clássico de Fanger (PMV) para predição da real sensação térmica nos profissionais intensivistas adaptados ao clima tropical, procurando identificar o limite de concordância entre tais métodos. As amostras foram coletadas em nove unidades de terapia intensiva (UTIs) públicas da cidade de João Pessoa/Paraíba/Brasil e as estimações obtidas pelos modelos foram confrontadas através da aplicação da análise de concordância entre métodos de Bland-Altman. Os testes identificaram que dentre os métodos selecionados o que apresentou menor viés para estimações da real sensação foi o PMV clássico se comparado aos modelos adaptativos indicados para indivíduos aclimatados a regiões quentes e úmidas características de climas tropicais. Entretanto, a amplitude do viés foi dependente da faixa de estimação, ou seja, à medida que as estimações se mantiveram entre valores de -1,5 e 0,2 o viés tendeu a ser minimizado. A análise de concordância mútua entre os testes mostrou que o PMV e o PMVak são equivalentes quando suas estimações se situam entre $-0,5$ e 0,5 , o que indica que o fator de ponderação para adaptabilidade proposto pelo modelo PMVak não foi o suficiente para prever a aclimatação na amostra considerada.

Elamara Marama Araujo Vieira Universidade Federal da Paraíba J oão Pessoa - PB - Brasil

J onhatan Magno Norte da Silva Universidade Federal de Alagoas Delmiro Gouveira - AL -Brasil

Wilza Karla dos Santos Leite Universidade Federal da Paraíba J oão Pessoa - PB - Brasil

Manoel Gerônimo Lino Torres Centro Universitário CESMAC Maceió - AL- Brasil

Luiz Bueno da Silva Universidade Federal da Paraíba J oão Pessoa - PB - Brasil

Recebido em 05/06/16 Aceito em 26/09/16
Palavras-chave: Modelos adaptativos. PMV. Climas quentes e úmidos. Unidades de terapia intensiva.

\section{Abstract}

The aim of this study was to investigate the behaviour of adaptive models of thermal comfort (aPMVy e aPMVk) as compared with the classical Fanger model $(P M V)$ for the prediction of the actual thermal sensation of intensivist professionals adapted to the tropical climate, seeking to identify the limits of agreement between such methods. The samples were collected at nine public Intensive Care Units (ICUs) in the city of João Pessoa, Paraiba, Brazil and the estimates obtained by the models were compared by applying the concordance between Bland-Altman methods. The tests revealed that, among the selected methods, the one that showed the least bias estimating the real sensation was the classic PMV, compared with adaptive models indicated for individuals acclimatized to hot and humid regions, which are characteristic of tropical climates. However, the amplitude of the bias depended on the estimation range, i.e., as the estimation was kept between -1.5 and 0.2 values, the bias tended to be minimized. The mutual correlation analysis between tests showed that the PMV and aPMVk are equivalent when their estimates are between -0.5 and 0.5, indicating that the weighting factor for adaptability offered by the aPMVk model was not enough to provide acclimatization in the sample considered.

Keywords: Adaptive models. PMV. Hot and humid climates. ICU. 


\section{Introdução}

O conforto térmico vem sendo mais profundamente estudado desde meados da década de 70 , e teve avanços significativos a partir dos estudos de P. O. Fanger. É definido como uma condição de satisfação relatada pelos usuários (AMERICAN..., 2013), ou seja, é uma condição subjetiva decorrente do ajustamento corporal, comportamental e expectativas pessoais, as quais resultam de interação dinâmica e complexa entre o homem e o ambiente.

A construção do estado da arte relativo ao conhecimento do conforto térmico vem sofrendo diversas alterações em função do tempo. O que se postula atualmente no meio científico é uma tentativa de atualizar a forma de avaliar o conforto térmico, atualmente baseada na ISO 7730, para uma avaliação mais centrada em populações específicas, já que as particularidades de cada população podem afetar significativamente o estado de conforto (KIM et al., 2015).

O modelo do balanço térmico proposto por Fanger em 1970 idealiza o indivíduo como um receptor passivo dos estímulos térmicos e se baseia no princípio de que os efeitos de um dado ambiente são mediados pelas forças físicas de troca de calor entre corpo e meio. Contudo, Brager e Dear (1998) elucidaram através de uma revisão sistemática as evidências científicas que embasaram a teoria adaptativa do conforto, que vem a complementar a teoria do balanço térmico de Fanger e trazer novas luzes ao conhecimento da interação entre o homem e o ambiente.

Inúmeras modelagens surgiram a fim de internalizar a teoria adaptativa aos preceitos iniciais de Fanger; e se tem estipulado que o modelo em vigência, adotado pela norma ISO 7730 (INTERNATIONAL..., 2005), apresenta vieses significativos e não comporta mais uma avaliação fidedigna de acordo com o atual estado do conhecimento e com a complexidade dos ambientes atuais. Sobre essa ótica, surge a discussão da necessidade de atualizações das normas e método de avaliação, tendo em vista que as especificidades da população estudada podem levar a resultado inconsistentes (DEL FERRARO et al., 2015; ZHOU et al., 2014).

Porém, se de fato existem discrepâncias significativas entre a predição adotada pela ISO 7730 (INTERNATIONAL..., 2005) e a real sensação, questiona-se: Como os modelos de abordagens adaptativas se comportam em relação às sensações subjetivas e em relação ao PMV?
Dentre os diversos ambientes em que se pode avaliar o ambiente térmico, destaca-se os ambientes hospitalares, em especial as unidades de terapia intensiva (UTIs), que por lidarem com a seguridade e mantenabilidade de vidas torna-se um ambiente crítico para investigações. Assim, tem-se como objetivo investigar o comportamento de modelos adaptativos de PMV em relação ao modelo clássico de Fanger para predição da real sensação térmica nos profissionais em UTIs adaptados ao clima tropical, procurando identificar o limite de concordância entre tais métodos.

\section{Materiais e métodos}

\section{Campo de pesquisa e amostra}

As coletas foram realizadas em nove UTIs públicas da cidade de João Pessoa/Paraíba/Brasil, entre os dias 20 de julho e 29 de agosto de 2015. As características de cada unidade de análise são destacadas no Quadro 1, ressaltando-se que toda a pesquisa foi realizada em ambientes climatizados artificialmente, que comumente mantinham um único condicionador de ar para toda a UTI, sendo nesse caso necessário manter velocidades do ar superiores às recomendações para atingir regiões mais distantes, ou, no caso de mais de um condicionador de ar, esses eram posicionados de maneira inadequada criando microrregiões térmicas.

As UTIs apresentam tamanhos e formatos arquitetônicos diversos, tendo em vista que não há uma padronização para tanto. Em todos os casos elas são localizadas ou no andar térreo ou no primeiro piso. Apresentam predominantemente iluminação artificial, com exceção das UTIs 5 e 6 , que possuem grandes janelas para iluminação natural, dispostas em diversos ângulos em relação à sala, de maneira que durante todo o dia a luz solar adentre ao recinto. São, porém, vedadas à ventilação natural. Tais setores apresentam no seu interior um grande quantitativo de aparato tecnológico de suporte à vida, localizados primordialmente próximo aos leitos de atendimento. No centro da sala, o assim nominado "posto de enfermagem", local onde os profissionais de saúde localizam-se para atividades administrativas, é a área com maior densidade de pessoas. A taxa de ocupação de tais unidades é diretamente proporcional à sua dimensão de espaço, tendo em vista que quanto maior a capacidade operacional maior a necessidade de profissionais para fornecimento do suporte assistencial. 
Quadro 1 - Características das UTIs avaliadas

\begin{tabular}{c|c|c|c|c|c}
\hline UTI & Especialidade & Data & $\mathbf{m}^{\mathbf{2}}$ & Iluminação & SAC \\
\hline 1 & Obstetrícia & 20 a 23 de ago. & 140,3 & Predominantemente artificial & Split \\
2 & Traumato-ortopedia & 24 a 26 de ago. & 110,2 & Predominantemente artificial & Split \\
3 & Geral & 29 a 31 de ago. & 251,8 & Artificial + natural & Central + Split \\
4 & Obstetrícia & 03 a 05 de set. & 112,2 & Predominantemente artificial & Split \\
5 & Traumato-ortopedia & 06 a 08 de set. & 141,6 & Predominantemente natural & Split \\
6 & Geral & 11 a 14 de set. & 78 & Predominantemente natural & Split \\
7 & Geral & 17 a 20 de set. & 114,7 & Artificial & Central \\
8 & Obstetrícia & 24 a 26 de set. & 116,8 & Artificial + natural & Central + Split \\
9 & Traumato-ortopedia & 27 a 29 de set. & 116,8 & Artificial + natural & Central + Split \\
\hline
\end{tabular}

Nota: ${ }^{*} \mathrm{SAC}=$ Tipo de sistema de condicionamento do ar.

A amostra agrega profissionais que compõem a equipe assistencial das UTIs investigadas, a saber: médico, enfermeiro, fisioterapeuta e técnico de enfermagem, tendo como critério de inclusão o aceite formal da participação na pesquisa e a permanência mínima por seis horas ininterruptas no setor. Os profissionais investigados possuem atribuições puramente assistenciais, ou seja, de suporte ao tratamento clínico, e por isso apresentam característica de trabalho semelhante, apresentando contato direto com o paciente e permanência contínua na UTI, tendo como atribuição específica do médico o diagnóstico e gerenciamento da assistência prestada, do enfermeiro o cuidado contínuo e direto ao paciente e acolhimento do familiar, do fisioterapeuta a reabilitação de diversos sistemas orgânicos e do técnico de enfermagem a prestação de cuidados básicos cotidiano tais como higienização, alimentação e medicação.

Os profissionais foram informados sobre o escopo da pesquisa e em seguida, em caso de aceite, foram orientados a assinar o termo de consentimento livre e esclarecido (TCLE).

O projeto de pesquisa foi previamente submetido ao Comitê de Ética em Pesquisa do Centro de Ciências da Saúde (CCS), vinculado à Universidade Federal da Paraíba (UFPB) e aprovado no dia 01/07/2015 sob o número CAEE 44388515.4.0000.5188.

\section{Procedimentos experimentais}

Para mensuração das variáveis térmicas utilizou-se o instrumento “TGD 400” (Instruterm). O aparelho de coleta foi calibrado, e, respeitando o tempo mínimo de adaptação de 30 minutos, foi então posicionado em pontos fixos previamente selecionados na sala, considerando o posicionamento dos condicionadores de ar, de maneira a não dificultar a mobilidade dos profissionais e não ficar próximo às portas, janelas ou condicionadores de ar. Para tanto, foram adotadas as recomendações de coleta indicadas na norma ISO 7730 (AMERICAN..., 2005) e ISO 7726 (2005).

As medições foram realizadas com intervalos de 1 minuto e os parâmetros classificados como grandezas envolvidas na avaliação do conforto térmico foram:

(a) temperatura de globo $\left({ }^{\circ} \mathrm{C}\right)$;

(b) temperatura de bulbo seco $\left({ }^{\circ} \mathrm{C}\right)$;

(c) temperatura de bulbo úmido $\left({ }^{\circ} \mathrm{C}\right)$; e

(d) umidade relativa do ar (\%).

Além de variáveis pessoais como taxa metabólica $\left(\mathrm{w} / \mathrm{cm}^{2}\right)$, isolamento térmico das vestimentas (clo) e sensação térmica autorreferida obtida através da questão "Com relação a sua sensação térmica, como você está se sentindo nesse momento?”, tendo como categorias de resposta as opções "Muito calor (+3)", "Com calor (+2)", "Levemente com calor (+1)", "Neutro (0)", "Levemente com frio (-1)", “Com frio (-2)” e "Muito frio (-3)”, conforme a ISO 10551 (INTERNATIONAL..., 2011).

Para obtenção dos índices PMV e PPD, foram considerados como taxa metabólica os valores de 1,2 met para os profissionais de UTIs localizadas nas dependências de maternidades, ou seja, com especialidade em obstetrícia, e 1,6 met para os profissionais das demais instituições apontadas no Quadro 1. Tal atribuição foi realizada considerando o nível da atividade de cada profissional do setor, tendo em vista que as maternidades (obstetrícia) possuem um menor fluxo de pacientes e consequentemente menor demanda ocupacional.

\section{Seleção dos modelos adaptativos analisados}

Para a seleção dos modelos adaptativos inicialmente realizou-se uma busca bibliográfica nas bases de dados "Web of Science" e "Science Direct" usando as palavras-chave "thermal comfort", "adaptation model" e "PMV", tendo como critérios de busca pertencer a um periódico 
com fator de impacto $\geq 2$, ano de publicação entre 2015 e 2016 e ser direcionado para avaliação de ambientes climatizados.

Associadamente, buscou-se selecionar modelos desenvolvidos a partir de análises com amostra adaptada às características climáticas similares às da cidade de pesquisa em questão, ou seja, climas quentes e úmidos. Os dois modelos mais adequados aos critérios de busca foram selecionados como apresentado na Tabela 1.

\section{Análise estatística}

Os dados obtidos em campo foram inicialmente tabulados em planilha eletrônica considerando o local e dia de coleta. Dividiu-se a análise dos dados em duas etapas: (1) preliminarmente a amostra foi caracterizada através de medidas de tendência central e de dispersão; e (2) em um segundo momento estimou-se o PMV pelo método clássico (ISO 7730 (AMERICAN..., 2005)) e pelos modelos adaptativos propostos por Kim et al. (2015) e Yang et al. (2015). Para estimação do modelo adaptativo PMVak obteve-se inicialmente o coeficiente de adaptabilidade $(\lambda)$ para ambientes quentes $\mathrm{e}$ também para ambientes frios, o que resultou, respectivamente, nos valores de $-0,02474$ e 0,45789. Comparou-se cada modelo individualmente com a percepção autorreferida de conforto dos profissionais intensivistas e os resultados obtidos por meio do PMV clássico e os modelos adaptativos foram confrontados através da aplicação da análise de concordância entre métodos de Bland-Altman.

O teste de Bland-Altman objetiva investigar o nível de concordância entre dois métodos de avaliação com fins similares, partindo-se das avaliações das diferenças entre duas variáveis $\left(d_{i}=X-Y\right)$, ou seja, do "viés" existente entre elas, da avaliação do valor médio $(\mathrm{X}+\mathrm{Y}) / 2)$ dessas mesmas variáveis e, finalmente, da dispersão dos pontos do viés $\left(\mathrm{d}_{\mathrm{i}}\right)$ ao redor da média, o que é então caracterizado como o erro (HIRATA; CAMEY, 2009).

A partir de tais valores obteve-se a diferença média das variáveis $(\bar{d})$ (Equação 1 ), que associada ao desvio padrão $\left(\mathrm{S}_{\mathrm{d}}\right)$ dá origem ao limite de concordância (Equação 2) entre as estimações dos modelos e a real percepção térmica.

$\bar{d}=\frac{\sum d_{i}}{n}$

Eq. 1

$\bar{d} \pm 1,96 S d$

Eq. 2

A diferença média entre as variáveis $(\bar{d})$ foi testada quanto à normalidade pelo teste de KolmogorovSmirnov (DANIEL, 2009), e a hipótese de o viés ser ou não igual a zero foi verificada pelo teste de Wilcoxon (DANIEL, 2009, p. 694) para duas amostras emparelhadas admitindo-se para ambos um nível de significância de $\alpha<0,05$. Os testes foram realizados no software $\mathrm{R}$ versão 3.2.4.

\section{Resultados e discussão}

\section{Caracterização da amostra}

Entrevistou-se um total de 128 profissionais, sendo 103 mulheres e 25 homens. A amostra contou com 10 médicos (7,8\%), 23 enfermeiros (18\%), 26 fisioterapeutas (26\%) e 69 técnicos de enfermagem (53\%). As características da amostra são apresentadas com mais detalhes na Tabela 2.

Tem-se uma amostra prevalentemente feminina, jovem e com características antropométricas associadas ao perfil de indivíduos saudáveis. Cabe salientar que as mulheres com idade inferior a 50 anos, semelhantemente ao perfil da amostra desta pesquisa, tendem a julgar $o$ ambiente mais severamente em comparação aos homens, ou seja, possuem uma zona de conforto mais restrita, tendo $12 \%$ a mais de probabilidade de se sentirem insatisfeitas com relação à temperatura (KIM et al., 2013; DASCALAKI et al. 2009).

Tabela 1 - Modelos selecionados

\begin{tabular}{c|c|c}
\hline Autor & Modelo & Abreviatura \\
\hline \multirow{2}{*}{ Kim et al. (2015) } & $P M V a=\frac{P M V}{1+\lambda \times P M V}$ & \\
& Onde: $\lambda=\frac{\Sigma(\mathrm{Yi}-\mathrm{Xi})}{n} \quad \mathrm{Xi}=\frac{1}{\text { Sensação }}$ e Yi $=\frac{1}{P M V}$ & \\
\hline Yang et al. (2015) & PMVa $=0,22 \mathrm{PMV}^{2}+0,45 \mathrm{PMV}-0,1$ & PMVay \\
\hline
\end{tabular}

Fonte: adaptado de Kim et al. (2015) e Yang et al. (2015). 
Tabela 2 - Perfil da amostra

\begin{tabular}{|c|c|c|c|c|c|c|}
\hline \multicolumn{2}{|c|}{ Item } & \multirow{2}{*}{$\begin{array}{c}\mathbf{n} \\
103 \\
25\end{array}$} & \multirow{2}{*}{$\begin{array}{c}\mathbf{\%} \\
80,5 \% \\
19,5 \% \\
\end{array}$} & \multirow[t]{2}{*}{ Média } & \multirow[t]{2}{*}{ Mediana } & \multirow[t]{2}{*}{ Desvio-padrão } \\
\hline Sexo & $\begin{array}{c}\text { Feminino } \\
\text { Masculino }\end{array}$ & & & & & \\
\hline \multicolumn{2}{|c|}{ Idade (Anos) } & & & 35,5 & 34 & $\pm 8,2$ \\
\hline \multicolumn{2}{|c|}{ Altura (Metros) } & & & 1,64 & 1,63 & $\pm 0,1$ \\
\hline \multicolumn{2}{|c|}{ Peso (Quilos) } & & & 72,4 & 70 & $\pm 18,2$ \\
\hline \multicolumn{2}{|c|}{ IMC* } & & & 26,8 & 25,6 & $\pm 5,6$ \\
\hline \multicolumn{2}{|c|}{ Tempo de serviço (Anos) } & & & 7,4 & 5 & $\pm 5,9$ \\
\hline
\end{tabular}

Nota: *IMC = Índice de Massa Corpórea.

\section{Conformação térmica das unidades de análise}

Obteve-se um total 12.021 medições, com médias e desvios padrões expostos na Tabela 3. Nos dados verificou-se os índices individuais de PMV, a partir dos quais foi possível fazer a média para cada UTI investigada.

Observa-se que a temperatura do ar nas UTIs apresentou-se em um intervalo entre $18 \%$ e $22^{\circ} \mathrm{C}$, com variações diárias inferiores a $2{ }^{\circ} \mathrm{C}$. Essas temperaturas, associadas à excessiva umidade relativa (\%), proporcionaram uma sensação térmica estimada (PMV) preferencialmente entre a neutralidade e a sensação levemente fria.

Ademais, a temperatura média radiante (TRM), por apresentar valores similares à temperatura do ar, demonstra a ausência de fontes significativas de radiação para geração de calor no ambiente. Podese, portanto, classificar as unidades de análise como ambientes termoneutros, porém a margem de padrões higiênicos estipulados para o local, considerando a umidade relativa, tendo em vista que em ambientes hospitalares a umidade relativa deve estar no intervalo entre 35 e 65\% (BRASIL, 2003).

As maiores variações de temperatura foram observadas nos setores onde houve, respectivamente, maior quantidade de iluminação natural, decorrente da influência da entrada de luz sob os parâmetros térmicos, seguida da UTI com maiores dimensões arquitetônicas e maior taxa de ocupação, que nesse caso é influenciada pelo fluxo de pessoas e pela capacidade insuficiente do sistema de condicionamento aéreo em compensar as dimensões do ambiente.

\section{Análise de concordância: método Bland-Altman}

Os parâmetros obtidos dessa análise estão expostos na Tabela 4. Previamente cabe identificar que os métodos avaliados são considerados equivalentes se as diferenças oferecidas nos limites de concordância $(\bar{d})$ são aceitáveis, admitindo-se o intervalo de valores $\pm 0,25$ (HUMPHREYS; NICOL, 2002).

Observa-se que dentre os modelos avaliados o PMV clássico adotado pela ISO 7730 se destaca por obter o menor viés, ou seja, $\bar{d}$ próximo a zero e não estatisticamente significativo em relação à percepção, como demonstrado pelo teste de Wilcoxon. Similarmente, o PMVak mostrou-se em concordância com a sensação autorreferida, fato não observado para o PMVay em decorrência de seu afastamento dos limites aceitáveis para concordância $( \pm 0,25)$.

A visualização das distinções entre os testes também pode ser identificada no intervalo de confiança, à medida que no PMV e PMVak tem-se valores positivos e negativos, o que denota que o real valor de $\bar{d}$ pode ser zero, entretanto os valores do intervalo de confiança do PMVay não oferecem tal possibilidade.

Cabe destacar que os três modelos possuem limites de concordância com margens muito amplas, o que indica que em casos extremos a percepção autorreferida pode estar cerca de duas vezes acima ou abaixo das estimações, com valores de desvio padrão e erro padrão similares entre si.

A Figura 1 expõe graficamente a aproximação do valor de $\bar{d}$ gerado para o teste entre PMV e a sensação autorreferida ao limite zero, mostrando o quanto o PMV clássico e a sensação autorreferida estão alinhados e também seus limites de concordância, podendo-se observar que o viés entre os testes diminuiu quando os valores das estimações se mantiveram preferencialmente entre -1,5 e 0,2, o que implica afirmar que na amostra considerada estimações do PMV clássico entre tais valores são mais fortemente condizentes com a real sensação térmica dos indivíduos, tendo assim uma medida de erro reduzida. Entretanto, à medida que as estimações se aproximaram dos valores mais extremos houve um aumento do viés entre os testes, consequentemente aumentando a tendência ao erro. 
Tabela 3 - Média e desvios padrões das variáveis térmicas

\begin{tabular}{c|c|c|c|c|c|c}
\hline UTI & Data & $\mathbf{T a}\left({ }^{\circ} \mathbf{C}\right)$ & $\mathbf{T g}\left({ }^{\circ} \mathbf{C}\right)$ & $\mathbf{T r m}\left({ }^{\circ} \mathbf{C}\right)$ & HR(\%) & PMV \\
\hline 1 & 20 a $22 / 07$ & $19,3 \pm 0,6$ & $19,4 \pm 0,7$ & $19,3 \pm 0,7$ & $84,8 \pm 1,2$ & $-1,25 \pm 1,1$ \\
2 & 24 a 26/07 & $22,9 \pm 0,3$ & $22,8 \pm 0,5$ & $22,9 \pm 0,4$ & $78,9 \pm 1,9$ & $-1,01 \pm 0,2$ \\
3 & 29 a 31/07 & $20,9 \pm 1,5$ & $21,2 \pm 1,6$ & $20,9 \pm 1,4$ & $77,6 \pm 3,7$ & $-0,47 \pm 0,4$ \\
4 & 03 a 05/08 & $20,3 \pm 0,2$ & $20,4 \pm 0,3$ & $20,3 \pm 0,2$ & $82,2 \pm 1,2$ & $-0,28 \pm 0,2$ \\
5 & 06 a 08/08 & $18,4 \pm 1,5$ & $20,4 \pm 1,7$ & $19,7 \pm 1,8$ & $82,4 \pm 6,5$ & $-0,85 \pm 0,4$ \\
6 & 11 a $14 / 08$ & $21,8 \pm 0,7$ & $22,1 \pm 1,1$ & $21,9 \pm 0,8$ & $81,1 \pm 3,3$ & $-0,08 \pm 0,6$ \\
7 & 17 a 20/08 & $22,5 \pm 0,6$ & $22,3 \pm 0,6$ & $22,5 \pm 0,7$ & $75,4 \pm 1,8$ & $-0,14 \pm 0,2$ \\
8 & 24 a $26 / 08$ & $22,8 \pm 1,2$ & $22,9 \pm 0,9$ & $22,8 \pm 1,2$ & $74,8 \pm 2,5$ & $0,01 \pm 0,3$ \\
9 & 27 a $29 / 08$ & $22,3 \pm 0,7$ & $21,9 \pm 1,1$ & $22,3 \pm 1,2$ & $74,1 \pm 2,7$ & $-0,15 \pm 0,2$ \\
\hline
\end{tabular}

Nota: Legenda:

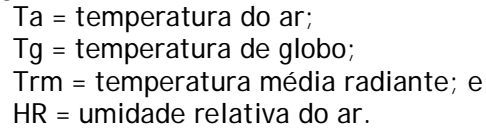

Tabela 4 - Teste de concordância entre modelos de conforto com a sensação autorreferida

\begin{tabular}{l|c|c|c}
\hline \multicolumn{1}{c|}{ Indicador } & PMV & PMVay & PMVak \\
\hline $\bar{d}$ & $-0,009$ & 0,261 & 0,142 \\
$\mathrm{IC}(\bar{d})$ & $(-0,232 ; 0,214)$ & $(0,045 ; 0,476)$ & $(-0,075 ; 0,359)$ \\
\hline $\mathrm{S}_{\mathrm{d}}$ & 1,276 & 1,235 & 1,244 \\
\hline Erro-padrão & 0,113 & 0,109 & 0,109 \\
\hline Teste de normalidade de $\bar{d}$ & 0,489 & $2,625 \times 10^{-05}$ & 0,385 \\
\hline Teste de Wilcoxon & $0,693^{* *}$ & $0,084^{* *}$ & $0,323^{* *}$ \\
\hline Limite inferior & $-2,512$ & $-2,161$ & $-2,297$ \\
IC & $(-2,898 ;-2,125)$ & $(-2,535 ;-1,786)$ & $(-2,673 ;-1,919)$ \\
\hline Limite superior & 2,493 & 2,683 & 2,581 \\
IC & $(2,106 ; 2,880)$ & $(2,308 ; 3,057)$ & $(2,204 ; 2,958)$ \\
\hline
\end{tabular}

Nota: Legenda:

IC = Intervalos de confiança; e

$* \mathrm{H}_{0}: \bar{d}=0$.

Figura 1 - Limite de concordância entre PMV e sensação autorreferida

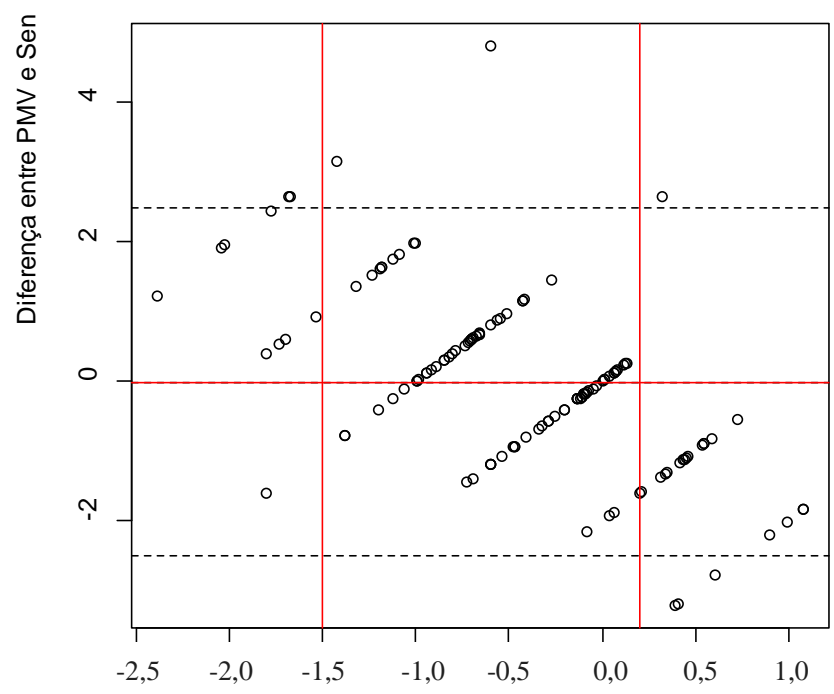

Nota: Legenda:

Média entre PMV e Sensaçao aı

linha vermelha horizontal: limite zero; e

linhas vermelhas verticais: valores mais próximos ao limite zero. 
As publicações atuais afirmam que o PMV superestima a sensação térmica real, especialmente em indivíduos adaptados ao clima quente e úmido, já que nesse grupo de indivíduos, em decorrência de mecanismos adaptativos, a neutralidade térmica não corresponderia ao conforto térmico propriamente dito, mas sim às sensações ligeiramente frias (YAO; LI; LIU, 2009; YANG et al., 2015; HWANG et al., 2009).

De fato, nesta pesquisa observa-se que as predições do PMV nos indivíduos adaptados a climas quentes e úmidos têm uma menor tendência ao erro quando o PMV está entre -1,5 e 0,2, o que pode indicar que a zona de conforto para essa amostra encontra-se nessa faixa de predições gerando estimações mais fidedignas às reais sensações.

Na Figura 2 a sensação autorreferida é comparada às estimações do modelo adaptativo proposto por Yang et al. (2015) (PMVay), podendo-se observar um modelo mais restritivo se comparado às estimações do modelo PMV clássico e um aumento do afastamento de $\bar{d}$ ao limite zero se comparado ao teste com PMV. Nessa nova conformação o viés é minimizado quando as estimações preveem valores entre -1 e 0,2 . Assim, quanto mais os valores se afastam dessa faixa maior o erro.

Finalmente, a Figura 3 compara as estimações do modelo adaptativo proposto por Kim et al. (2015) (PMVak) à sensação autorreferida, em que se pode observar similaridades com as estimações feitas pelo PMV clássico, inclusive quanto à margem das estimações na qual o viés é reduzido, mantendo-se entre $-1,5$ e 0,2 nesse modelo.

Dentre os três modelos avaliados o que melhor correspondeu a verdadeiras sensações dos profissionais foi ainda o modelo clássico do PMV adotado pela ISO 7730 e proposto por Fanger em 1970, mantendo um menor viés. No PMVay as diferenças oferecidas nos limites de concordância $(\bar{d})$ foram aceitáveis, porém com menor poder se comparado ao PMV clássico, enquanto o fator de ponderação para a adaptabilidade proposto pelo modelo PMVak não foi capaz nessa amostra de proporcionar estimações mais fidedignas do que os demais modelos estudados e pelo que pode requerer adequações.

Portanto, na amostra adaptada a climas quentes e úmidos os modelos de PMV clássico e adaptativo seriam precisos apenas em condições aproximadas de conforto, que está relacionada às sensações levemente frias, o que implica afirmar que os ocupantes desenvolveram mecanismos adaptativos, desconsiderados pela predição clássica, mas também pelas predições sugeridas nos modelos adaptativos, indicando a necessidade de reformulações da amplitude da zona de conforto direcionada para as regiões climáticas em questão.

Ao identificar a aderência da predição de cada modelo com a real sensação térmica cabe analisar a aderência mútua entre os testes. Essa análise é realizada nas seções subsequentes buscando identificar possíveis similaridades e discrepâncias.

Figura 2 - Limite de concordância entre PMVay e sensação autorreferida

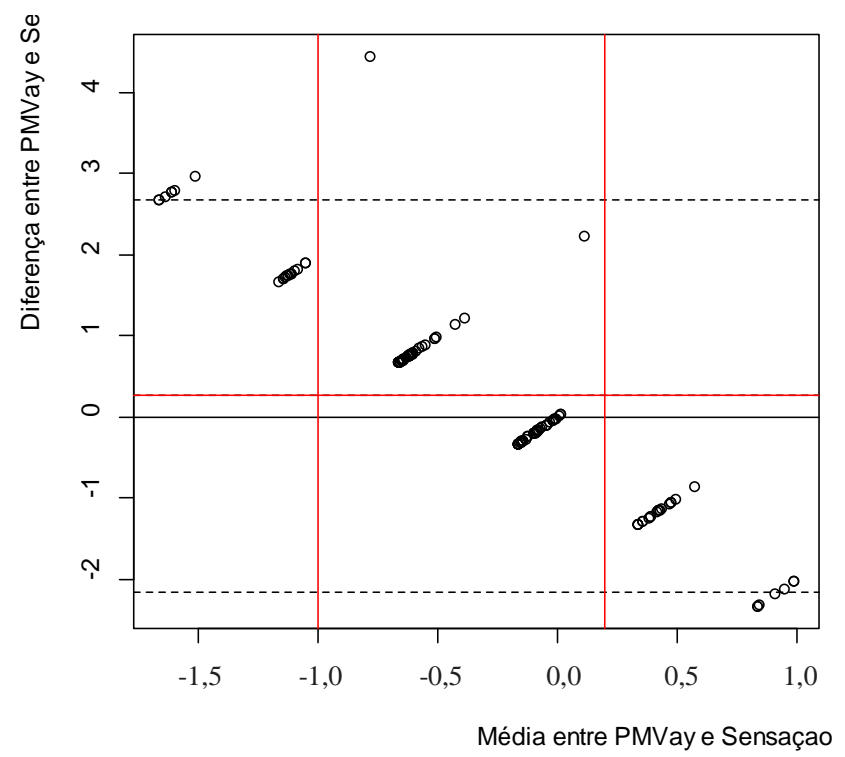

Nota: Legenda:

linha vermelha horizontal: valor de $\bar{d}$; e

linhas vermelhas verticais: valores mais próximos ao limite zero. 
Figura 3 - Limite de concordância entre PMVak e sensação autorreferida



Nota: Legenda:

linha vermelha horizontal: valor de $\bar{d}$; e

linhas vermelhas verticais: valores mais próximos ao limite zero.

Tabela 5 - Teste de concordância entre modelos PMV e PMVak

\begin{tabular}{l|c|c}
\hline \multicolumn{1}{c|}{ Indicador } & Teste & Intervalos de confiança \\
\hline $\bar{d}$ & $-0,151$ & $(-0,190 ;-0,112)$ \\
$\mathrm{S}_{\mathrm{d}}$ & 0,221 & \\
Erro-padrão & 0,019 & \\
Teste de normalidade de $\bar{d}$ & $2,2 \times 10^{-16}$ & \\
Teste de Wilcoxon & $2,2 \times 10^{-16 *}$ & \\
Limite inferior & $-0,586$ & $(-0,653 ;-0,518)$ \\
Limite superior & 0,283 & $(0,215 ; 0,349)$ \\
\hline
\end{tabular}

Nota: $* \mathrm{H}_{0}: \bar{d}=0$.

\section{PMV e PMVak}

A Tabela 5 expõe os indicadores de nível de concordância entre métodos PMV e PMVak. Observa-se que apesar de o teste de Wilcoxon indicar que existe diferença entre as estimações feitas pelos testes, os valores de $\bar{d}$ mantiveram-se dentro dos limites aceitáveis $( \pm 0,25)$, o que indica que são similares em suas predições, ou seja, os métodos são equivalentes.

Ademais, se comparado aos parâmetros prévios, as estimações feitas pelos testes PMV e PMVak possuem uma menor dispersão em relação à média $\left(\mathrm{S}_{\mathrm{d}}\right)$, menor erro padrão e valores de limites de concordância mais aproximados ao zero, denotando que podem gerar estimações mais aproximadas entre si do que com a sensação autorreferida.

A Figura 4 expõe graficamente os dados apresentados na Tabela 5, na qual se observa que a igualdade entre os métodos foi potencializada a determinadas estimações. Quando as estimações se mantiveram entre os valores de -0,5 e 0,5 (linhas verticais) formou-se um platô não viesado, ou seja, com diferenças nulas, o que indica que em estimações a tais dimensões a diferença entre os métodos é zero. Tem-se que quanto mais a estimação de ambos os métodos se aproxima de zero maior suas similaridades, entretanto 0 afastamento desse valor resulta em um aumento exponencial em suas diferenças.

Assim, tem-se que o modelo proposto por Kim et al. (2015), aqui intitulado PMVak, é eficaz para predição da sensação subjetiva no grupo estudado, porém é similar ao PMV clássico e apresentou-se pouco sensível a compensação das lacunas relativas às questões adaptativas da amostra. 


\section{PMV e PMVay}

A Tabela 6 expõe os indicadores de nível de concordância entre métodos PMV e PMVay. Observa-se que nesse novo contexto o $\bar{d}$ foi superior aos limites aceitáveis de concordância $( \pm 0,25)$ e estatisticamente diferente de zero, como mostrado pelo teste de Wilcoxon, podendo interpretá-los como modelos com predições distintas entre si.
A maior diferença entre os dois testes $(\bar{d})$, se comparado aos dados prévios, resultou em uma redução na amplitude dos valores de estimação em que os testes se equiparam, ficando reduzida a valores entre -0,5 e 0,2 (linhas verticais), como indicado na Figura 5. Assim, nesta faixa de valores de estimação os testes apresentam as maiores similaridades.

Figura 4 - Limite de concordância entre PMV e PMVak

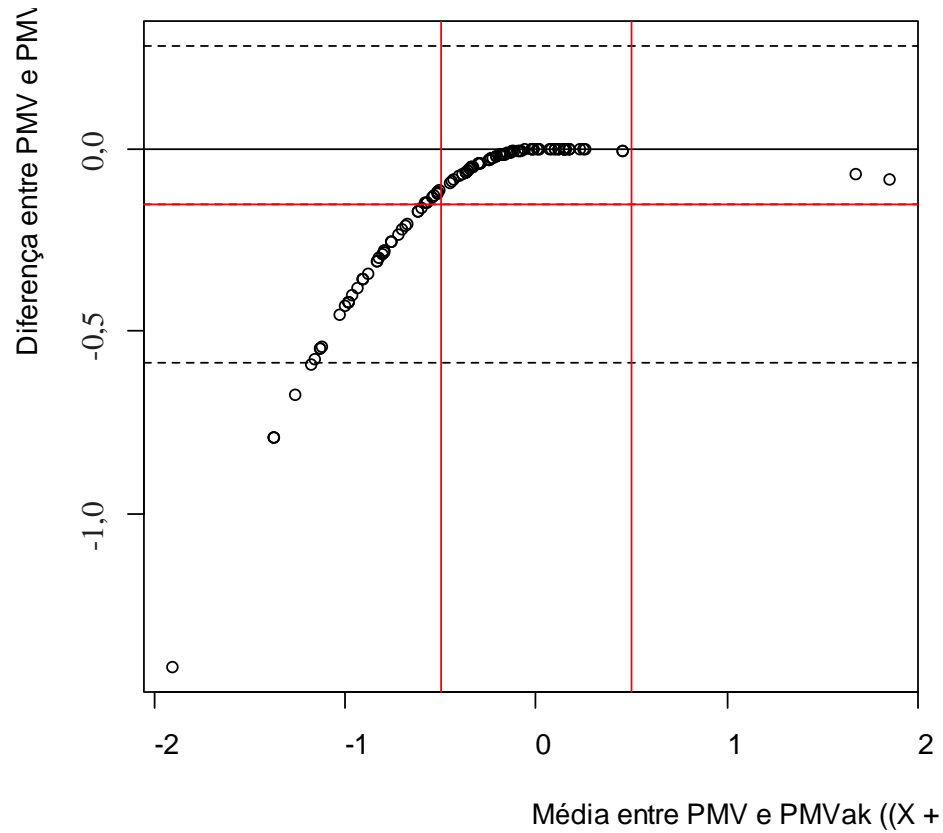

Nota: Legenda:

linha vermelha horizontal: valor de $\bar{d}$; e

linhas vermelhas verticais: valores mais próximos ao limite zero.

Tabela 6 - Parâmetros do teste entre modelos PMV e PMVay

\begin{tabular}{l|c|c}
\hline \multicolumn{1}{c|}{ Indicador } & Teste & Intervalos de confiança \\
\hline $\bar{d}$ & $-0,2701$ & $(-0,355 ;-0,185)$ \\
$\mathrm{S}_{\mathrm{d}}$ & 0,487 & \\
Erro-padrão & 0,0431 & \\
Teste de normalidade de $\bar{d}$ & $2,22 \times 10^{-16}$ & \\
Teste de Wilcoxon & $9,155 \times 10^{-08^{*}}$ & $(-1,372 ;-1,0773)$ \\
Limite inferior & $-1,225$ & $(0,537 ; 0,832)$ \\
Limite superior & 0,684 & \\
\hline
\end{tabular}

Nota: $* \mathrm{H}_{0}: \bar{d}=\overline{0}$. 
Figura 5 - Limite de concordância entre PMV e PMVay

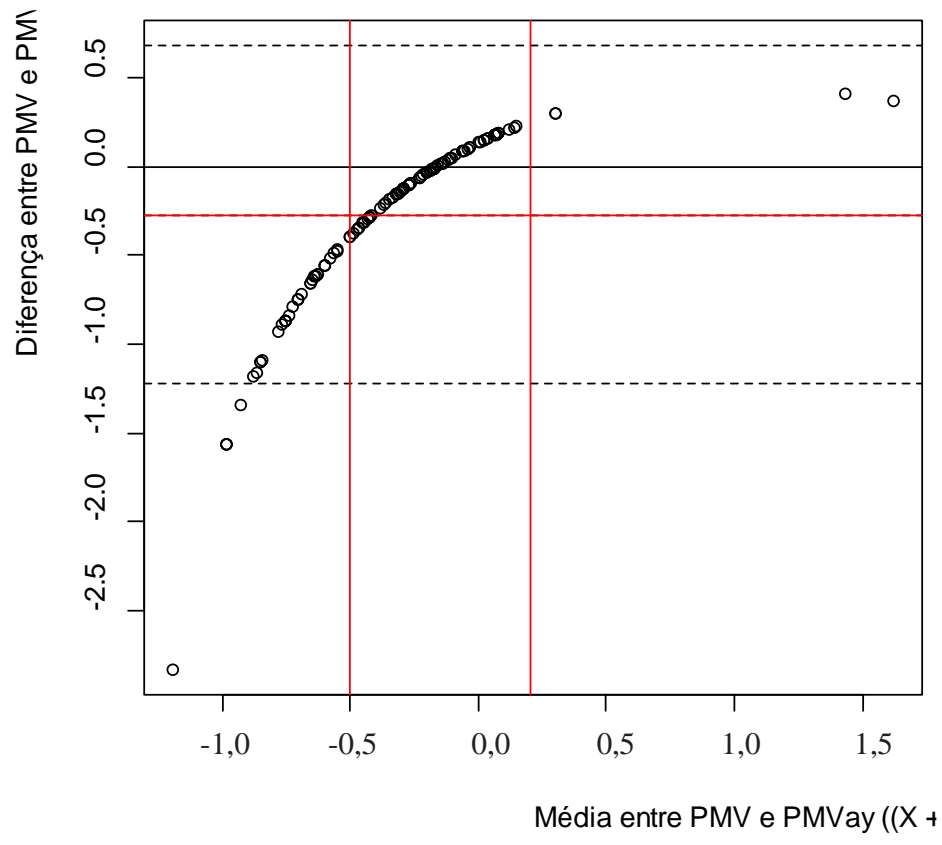

Nota: Legenda:

linha vermelha horizontal: valor de $\bar{d}$; e

linhas vermelhas verticais: valores mais próximos ao limite zero.

Portanto, apesar das tentativas de suprir possíveis lacunas deixas pelo PMV clássico, integrando sua avaliação em populações e situações específicas, como no caso de intensivistas aclimatados em regiões quentes e úmidas e inseridos em ambientes artificialmente climatizados, as estimações dos modelos adaptativos propostos, na amostra considerada, mostraram-se demasiadamente similares as estimações fornecidas pelo PMV clássico adotado pela ISO 7730 (INTERNATIONAL..., 2005).

Essas similaridades foram especialmente presentes a determinadas faixas de estimações, consideradas como faixas de conforto para o perfil climático considerado (YAO et al., 2009; YANG et al., 2015; HWANG et al., 2009). Porém, na previsão da real sensação dos indivíduos os três métodos considerados apresentaram inadequações nas estimativas localizadas à margem dessa faixa de conforto, devendo requer maiores esforço para compensá-las.

\section{Conclusões}

Objetivando-se entender como os novos modelos propostos para funções adaptativas se comportam frente às estimações da sensação real e também frente ao PMV clássico, considerando indivíduos adaptados a climas quentes e úmidos expostos a ambientes climatizados artificialmente, realizou-se uma análise de concordância pelo método de BlandAltman.

Identificou-se que dentre os métodos selecionados o que apresentou menor viés para estimações da real sensação foi o PMV clássico se comparado aos modelos adaptativos indicados para indivíduos aclimatados a regiões quentes e úmidas, como a cidade de João Pessoa, PB. Entretanto, a amplitude do viés foi dependente da faixa de estimação, ou seja, à medida que as estimações se mantiveram entre valores de -1,5 e 0,2 o viés tendeu a ser minimizado.

A análise de concordância mútua entre os testes mostrou que o PMV e o PMVak são equivalentes quando suas estimações se situam entre -0,5 e 0,5, o que indica que o fator de ponderação para adaptabilidade $(\lambda)$ proposto pelo modelo PMVak não foi o suficiente para prever a aclimatação na amostra considerada, enquanto o PMV e o PMVay mostraram-se distintos, porém com estimações aproximadas em uma pequena faixa de predições (entre -0,5 e 0,2). Ademais, observando o desvio padrão, o erro padrão e os limites de concordância inferior e superior, modelos PMV e PMVak geram estimações com uma maior precisão mútua do que em relação à sensação autorreferida.

Entende-se que apesar dos esforços para compensar o peso da adaptabilidade de grupos expostos a situações térmicas diversas, na amostra considerada as estimações resultantes de modelos distintos 
apresentam-se similares entre si e ainda com falhas para prever sensações mais extremas necessitando de maiores investigações na tentativa de aperfeiçoar tais métodos e ajustá-los às populações específicas.

\section{Referências}

AMERICAN SOCIETY OF HEATING, REFRIGERATING AND AIR-CONDITIONING ENGINEERS. ASHRAE 55: thermal environmental conditions for human occupancy. Atlanta, 2013.

BRAGER, G. S.; DEAR, R. J. Thermal Adaptation in the Built Environment: a literature review. Energy and Buildings, v. 27, n. 1, p. 83-96, 1998. BRASIL. Resolução - RE $\mathbf{n}^{\mathbf{0}}$ 9, de 16 de janeiro de 2003. Agência Nacional de Vigilância Sanitária, 2003.

DANIEL, W. W. Biostatistics: a foundation for analysis in the health sciences. $9^{\text {th }}$ ed. New Jersey: John Wiley \& Sons, 2009.

DASCALAKI, E. G. et al. Indoor Environmental Quality in Hellenic Hospital Operating Rooms. Energy and Buildings, v. 41, n. 5, p. 551-560, 2009.

DEL FERRARO, S. et al. A Field Study on Thermal Comfort in an Italian Hospital Considering Differences in Gender and Age. Applied Ergonomics, v. 50, p. 177-184, set. 2015.

HIRAKATA, V.N.; CAMEY, A.S. Análise de Concordância Entre Métodos de Bland-Altman. Revista do Hospital de Clínicas de Porto Alegre, v. 29, n. 3, p. 261-268, 2009.

HUMPHREYS, M.; NICOL, J. F. The Validity of ISO-PMV for Predicting Comfort Votes in EveryDay Thermal Environments. Energy and Buildings, v. 34, p. 667-684, 2002.
HWANG, R.-L. et al. Thermal Perceptions, General Adaptation Methods and Occupant's Idea About the Trade-Off Between Thermal Comfort and Energy Saving in Hot-Humid Regions.

Building and Environment, v. 44, n. 6, p. 11281134, 2009.

INTERNATIONAL ORGANIZATION FOR STANDARDIZATION. ISO 10551: ergonomics of the thermal environment: assessment of the influence of the thermal environment using subjective judgement scales. Genebra, 2011.

INTERNATIONAL ORGANIZATION FOR STANDARDIZATION. ISO 7726: ergonomics of the thermal environments: instruments for measuring physical quantities. Geneva, 2005.

INTERNATIONAL ORGANIZATION FOR STANDARDIZATION. ISO 7730: ergonomics of the thermal environment: analytical determination and interpretation of thermal comfort using calculation of the PMV and PPD indices and local thermal comfort criteria. Geneva, 2005.

KIM, J. T. et al. Development of the Adaptive PMV Model for Improving Prediction

Performances. Energy and Buildings, v. 98, p. 100-105, 2015.

KIM, J. et al. Gender Differences in Office Occupant Perception of Indoor Environmental Quality (IEQ). Building and environment, v. 70, p. 245-256, 2013.

YANG, Y. et al. A Study of Adaptive Thermal Comfort in a Well-Controlled Climate Chamber. Applied Thermal Engineering, v. 76, p. 283-291, 2015.

YAO, R.; LI, B.; LIU, J. A Theoretical Adaptive Model of Thermal Comfort: Adaptive Predicted Mean Vote (aPMV). Building and Environment, v. 44, n. 10, p. 2089-2096, 2009.

ZHOU, X. et al. A Model for Predicting Thermal Sensation of Chinese People. Building and Environment, v. 82, p. 237-246, 2014. 


\section{Elamara Marama Araujo Vieira}

Departamento de Estatística, Centro de Ciencias Exatas e da Natureza | Universidade Federal da Paraíba | Campus I, Castelo Branco J oão Pessoa - PB - Brasil | CEP 58051-900 | Tel.: (83) 3216-7124 | E-mail: elamaravieira@gmail.com

\section{J onhatan Magno Norte da Silva}

Departamento de Engenharia de Produção | Universidade Federal de Alagoas | Rodovia AL 145, Km 3, no 3849, Campus Sertão, Cidade Universitária | Delmiro Gouveira - AL -Brasil | CEP 57480-000 | Tel.: (82) 3214-1749 | E-mail: jonhatanmagno@hotmail.com

\section{Wilza Karla dos Santos Leite}

Departamento de Psicologia, Programa de Pós-Graduação em Psicologia Social | Universidade Federal da Paraíba | Campus I| J oão Pessoa - PB - Brasil | CEP 58051-900 | Tel.: (83) 3216-7000| E-mail: wilzakarlas@yahoo.com.br

\section{Manoel Gerônimo Lino Torres}

Departamento de Engenharia de Produção | Centro Universitário CESMAC | Rua Capitão Samuel Lins, s/n, Farol | Maceió - AL- Brasil | CEP 57021-140 | Tel.:: (82) 3215-5042 | E-mail: manoel.torres@hotmail.com

Luiz Bueno da Silva

Departamento de Engenharia de Produção | Universidade Federal da Paraíba | Campus I, Castelo Branco | J oão Pessoa - PB - Brasil | CEP 58051-900 | Tel.: (83) 3216-7124 | E-mail: silvalb@superig.com.br

\section{Revista Ambiente Construído}

Associação Nacional de Tecnologia do Ambiente Construído

Av. Osvaldo Aranha, 99 - 3o andar, Centro

Porto Alegre - RS - Brasil

CEP 90035-190

Telefone: +55 (51) 3308-4084

Fax: +55 (51) 3308-4054

www. seer. ufrgs. br/ ambienteconstruido

E-mail: ambienteconstruido@ufrgs.br 